\title{
Updates in the Prevention of Preeclampsia, What's Beyond Aspirin?
}

\author{
Abanoub Gabra ${ }^{1 *}$ and Mariam Gabra ${ }^{2}$ \\ ${ }^{1}$ Department of Obstetrics and Gynecology, Assuit University, Egypt \\ ${ }^{2}$ Nursing student, Hillsborough Community College, USA
}

*Corresponding author: Abanoub Gabra, Department of Obstetrics and Gynecology, Assuit University, Egypt.

Received Date: May 15, 2020

Published Date: June 02, 2020

\begin{abstract}
Hypertensive disorders of pregnancy are among the most common medical problems during pregnancy and they are associated with significant mortality and morbidity rate. Low dose Aspirin is already approved by many societies like ACOG and WHO to be used as prophylaxis for preeclampsia in high-risk patients. Recent studies showed a possible reduction in the incidence of preeclampsia and intrauterine growth restriction for high-risk mothers who taking LMWH during pregnancy. Although, the published evidence supporting LMWH is characterized by profound heterogeneity and inconsistency in terms of selection criteria and treatment regimens. Antepartum treatment with a combination of LMWH with low-dose ASA is endorsed by the American College of Chest Physicians and The American College of Obstetricians and Gynecologists for treatment of Antiphospholipid syndrome during pregnancy. WHO recommends Calcium as the first nutritional supplementation to prevent preeclampsia among population with low calcium in the diet. Folic acid and statins showed possible reduction in incidence of preeclampsia in high-risk patients but there is a need for further studies to confirm that. Dietary and lifestyle interventions have the potential to reduce the risk of preeclampsia. Both Metformin and vascular endothelial growth factors has promising preventive role that has been found through recent studies.
\end{abstract}

Keywords: Preeclampsia; Prevention; Hypertensive disorders of pregnancy

\section{Introduction}

Hypertensive disorders of pregnancy are among the most common medical problems during pregnancy and they are associated with significant mortality and morbidity rate [1]. They affect $4 \%$ of pregnant women and may cause serious complications like stroke, heart failure, and renal failure [2]. Those diseases are among the top 6 causes of maternal mortality in the USA being responsible for $10 \%$ of maternal deaths [3]. They should be considered as a syndrome rather than a single disease entity [4]. Highest mortality rates were found to be related to eclampsia, HELLP syndrome, hemorrhage, delayed diagnosis [5]. The incidence may be affected by parity, socioeconomic level, race, and environmental factors [6]. African Americans have a high incidence of preeclampsia, eclampsia, related maternal mortality [5]. Their incidence has been dramatically increased recently with significant health burden in terms of affection of both mother and fetus health [3]. Having standardized health care for those patients is associated with a significant reduction in both mortality and morbidity $[7,8]$.

Many societies contribute to Classifying hypertensive disorders of pregnancy in order to determine proper management lines and timelines for each category.

Chronic hypertension is defined as systolic BP of 140 or greater Or diastolic BP of 90 or greater Or both on 2 separate occasions at least 4 hours apart, This condition is diagnosed at or before 20 gestational weeks or already documented before pregnancy [9]. However, ACOG suggests that gestational hypertension or earlyonset preeclampsia should be considered if 1st trimester BP measures are within normal range [10]. Gestational hypertension 
is defined as elevated BP above 140/90 mmHg after 20 weeks at two separate occasions 6 hours apart in the absence of features of severe preeclampsia [11]. Severe gestational hypertension is defined by sustained elevated BP at more than or equal 160/110mmHg [12]. SOMANZ defines preeclampsia with severe features as a unique condition of pregnancy with multisystem effects involving liver, kidney and hematological parameters [13]. ACOG criteria for diagnosis include new-onset hypertension after 20 weeks associated with new-onset proteinuria $(>300 \mathrm{mg} / 24$ hours urine collection) [14]. In absence of proteinuria, diagnosis can be made upon presence of gestational hypertension plus any of the following: low platelet count less than $100.000 / \mathrm{cc}$, creatinine level more than 1.1 (double the baseline creatinine level in absence of other renal problem), raised liver enzymes (double baseline), pulmonary edema, visual or cerebral symptoms [15].

Low dose Aspirin is already approved by many societies like ACOG and WHO to be used as prophylaxis for preeclampsia in high-risk patients. In this article, we are going beyond Aspirin to know more updates about other possible prophylactic measures of preeclampsia including Low molecular weight heparin, Calcium, vitamin D, Arginine, statins, and Folic acid.

\section{Pathogenesis}

The pathogenesis of preeclampsia is not completely understood despite extensive researches focused on it $[16,17]$. Placental ischemia remains the most accepted theory that was postulated to explain the pathogenesis of preeclampsia because delivery of the fetoplacental unit remains the main curative line of treatment $[17,18]$. In addition to that, placental ischemia also explains other complications e.g. IUGR and oligohydramnios. Also, it explains a higher incidence of disease in patients with chronic hypertension, DM and autoimmune diseases. Placental ischemia may explain the effectiveness of both low dose Aspirin and Low molecular weight heparin $[19,20]$. In normal pregnancy, invasion of uterine arteries to cytotrophoblast causes their transformation from epithelial to endothelial cells with low resistance pressure allowing enough blood supply to fetus through a process called "pseudovasculogenesis" [18]. Cytotrophoblast cells initiate migration of extra villous trophoblast to decidua of uterus and invade partially myometrium inducing remodeling of spiral arteries [16]. 2-stage theory has been hypothesized recently to understand this pathology $[17,21]$. The first stage is abnormal events during embryogenesis of trophoblast which contribute to fetoplacental oxidative distress and abnormal release of antiangiogenetic factors in maternal circulation and subsequent multisystem endothelial dysfunction $[17,22]$. Abnormal remodeling of spiral arteries and early immunologically mediated events are considered major causes of those events [18]. Moreover, trophoblast fails to adequately invade uterine wall and spiral arteries so subsequently vascular resistance in this area could not be decreased to allow adequate placental transfusion [23]. Also, the failure of obliteration of tunica media of myometrium vessels contributes to inability of placenta to accommodate enough blood supply due to lack of thinning of those vessels [17]. This leads to excessive secretion of sFlt-1 (soluble-fms like tyrosine kinase-1) and soluble endoglin [24]. sFlt-1 binds in the blood to both the vascular endothelial growth factor (VEGF) and the placental growth factor (PLGF). Both sFlt-1 and low VEGF/PLGF play a major role in the development of systematic hypertension [21,24]. Later on, maternal syndrome may occur in terms of vascular endothelial dysfunction, intravascular hypercoagulability, and vasospasm leading to multiple systems dysfunctions [21]. Abnormal vascular changes in placenta are confirmed by histopathological examination of postpartum specimens of placenta which showed vascular infarcts and sclerosis of arterioles [17]. Immunological maternal reaction towards fetal and paternal derived Antigens may also contribute, which is considered a certain type of immunological intolerance [25]. Immunological theory is supported by high serum level of cell-free fetal DNA. This theory has been also postulated to understand pathogenesis of hyperemesis gravidarum [26,27]. Recently genetic factors were found to contribute to preeclampsia; Angiotensinogen gene T235 and Leiden factor deficiency were found to be associated with disease [28]. Also, the higher incidence of preeclampsia was found in trisomy 13 pregnancy than pregnancy with normal karyotyping [29,30]. Interestingly, the gene for sFlt-1 which is known for contributing to preeclampsia is also encoded in chromosome 13q [31].

\section{Low molecular weight Heparin (LMWH)}

Recent studies have shown potential reduction in the incidence of preeclampsia and IUGR for high-risk mothers who take LMWH during pregnancy [32]. Since preeclampsia has been hypothesized to be linked to thrombotic and vasoconstrictive events in placenta, It is proposed that LMWH has beneficial effects on certain patients [33]. Examination of the placenta of patients with preeclampsia or restriction of fetal growth after delivery revealed ischemic thrombotic lesions (33). Another research investigated the impact of LMWH on both in vitro and in vivo endothelial functions and reported that pregnant women at high risk of preeclampsia had major cardiovascular abnormalities relative to low-risk women at 24 weeks of gestation, validated by in vivo and in vitro tests [34]. They also reported with proof that LMWH affects the endothelial function and serum level of angiogenic proteins in pregnant women at elevated risk of preeclampsia. In parallel, LMWH also influenced the in vitro endothelial cell function, with significant proangiogenic reactions [34]. Researchers also investigate the effects of low molecular weight heparins (LMWHs) on the invasiveness of extra villous trophoblast and its expression of heparin bindingEGF and cysteine-rich angiogenic inducer 61 (Cyr61) related to the trophoblast invasion process [35], They found that LMWHs are capable of promoting trophoblast development and invasion because they are capable of stimulating the invasive extra villous trophoblast properties that provide a possible biological rationale for the clinical use of LMWH for placental-mediated complications of pregnancy not related to thrombophilia [35]. In addition, low- 
molecular heparin (LMWH) may control invasiveness and placental development of matrix metalloproteinase's (MMPs) and other tissue inhibitors in vitro trophoblast (36). Heparin greatly improved pro-MMPs and active forms, as well as the invasiveness of extra villous trophoblast and choriocarcinoma cells [37]. Heparin also blocked apoptosis caused by other agents including Staurosporin, kinase inhibitor of broad range, and Thrombin. In addition, Heparin decreased caspase-3 function, a hallmark of apoptosis in first trimester villous and extra villous trophoblast cell lines in human studies [37].

Another systematic study and meta-analysis of randomized controlled trials (RCTs) was undertaken, pregnant women were randomized to receive LMWH or non-fractionated heparin in addition to low-dose aspirin (LDA) and were contrasted with those receiving low-dose aspirin alone [38]. They concluded that the introduction of LMWH to low-dose aspirin in high-risk patients could decrease the prevalence of preeclampsia and small for gestational-age disease. Though they noted that these findings are focused on minimal data and require additional studies to validate this correlation [38]. The advantages of applying LMWH to Low dose Aspirin may be restricted to early onset of Preeclampsia, which is primarily attributed to an abnormal placentation [39]. Nearly all studies examined the relationship between preeclampsia and small-for-gestational-age infants as secondary outcomes so that evidence contained in such studies should be carefully considered [33].

In the other side, there is insufficient data based upon LMWH as a solo preventive tool. pregnant women with thrombophilia at an increased risk of venous thromboembolism or with previous placenta-mediated pregnancy complication, were registered in an open label randomized trial conducted in 36 tertiary care centers in five countries. Eligible participants obtained either Dalteparin Antepartum prophylactic dose once daily up to 20 weeks gestation, and twice daily up to at least 37 weeks gestation afterwards, or to no Dalteparin Antepartum (control group) [40]. Results revealed that Antepartum prophylactic Dalteparin does not prevent venous thromboembolism, pregnancy loss or placenta-mediated complications in women with thrombophilia, and is correlated with an increased risk of mild bleeding [40].

The published evidence supporting LMWH is characterized by a deep heterogeneity and inconsistency with regard to selection criteria and treatment regimens [38]. Obstetric or medical history in such preventive trials is usually the only entry criteria [38]. Most of the studies carried out have several limitations [41]. Several are underpowered or have a rather sluggish recruiting rate; some have taken patients with heterogeneous medical records into account [41]. Although for Anti-phospholipids syndrome, Antepartum treatment with a combination of LMWH with low-dose ASA is endorsed by the American College of Chest Physicians and The American College of Obstetricians and Gynecologists [41].

\section{Calcium supplements and vitamin D}

WHO recommends calcium as the first dietary supplement to avoid preeclampsia in low-calcium diet communities [42]. Other professional societies state recommend that as well $[43,44]$. This strong recommendation is focused on moderate-quality data from meta-analysis of randomized clinical trials, which show that calcium supplementation prevents around half of cases of preeclampsia [45]. The guideline recommends routine administration of 1,5-2,0 $\mathrm{g}$ of supplementary calcium beginning at $20 \mathrm{wk}$ gestation, and points out that separating calcium and iron supplementation by many hours is preferred in order to reduce the potential adverse effects of calcium on iron absorption [45].

The recommended calcium dosage recommended by the WHO, 1.5-2.0 g elemental calcium / day, is higher than both the current average requirement in the US and the recommended dietary dose for pregnant women (800 and $1000 \mathrm{mg}$, respectively) [45,46]. Dose of calcium determines the amount absorbed; as the dose's calcium concentration rises, the calcium 's fractional absorption reduces. Doses of $500 \mathrm{mg}$ are indicated per administration [46]. So, the WHO recommendations will involve 3 pill-taking events daily which may be inconvenient for patients [45]. Several calcium formulations are currently available in a variety of doses [47]. Compared with calcium gluconate, both calcium citrate and calcium carbonate are extremely bioavailable sources [47].

Iron and folic acid supplementation as part of routine antenatal treatment has been advised, for example to improve maternal and neonatal outcomes, to improve pregnancy anemia and to prevent neural tube defects especially in high-risk patients [14]. Calcium has been documented to interfere with iron absorption in vitro and short-term studies, but the clinical consequences of the interaction are limited over longer periods, since adaptive responses in iron regulatory mechanisms may resolve short-term interactions [45]. If held up to $500 \mathrm{mg}$ of elemental calcium / dose, the WHO recommended 3 daily doses. Separation of calcium and iron supplements will involve at least four different doses a day; this may lead to growing complexity of medicine intake with a possible negative effect on patient adherence [45]. Trials do nnot support the value of removing calcium from iron, because it is also speculative. In addition, the impact of the complexity of dosing would likely outweigh it to improve patient adherence. Therefore, It is recommended that women will take iron with one of the calcium doses, either in the morning or in the evening [42]. The WHO recommends that calcium supplementation be initiated at a gestational age of 20 weeks, based on the reference timing used in the meta-analyses on which the guidelines are based [45]. Although, further research is warranted to clarify research issues related to minimum effective dose, timing of initiation, mode of administration of prenatal calcium supplements and possible side effects [42]. 


\section{Arginine}

Pathogenesis of preeclampsia could be explained by endothelial dysfunction, platelet aggregation, and systemic vasoconstriction $[17,23]$. Nitric oxide is considered to be a potent inhibitor of platelet aggregation and vasoconstriction that participates in hemodynamic changes in preeclampsia patients [17]. Biochemical reactions involving L-Arginine as a producer of that substance produce nitric oxide. In preeclampsia patients, there is an increase in the synthesis of asymmetric dimethylarginine (ADMA), an endogenous inhibitor of nitric oxide synthase (NOS), and as a result, NO synthesis is reduced [48]. So researchers postulate that L- Arginine could antagonize ADMA to produce more Nitric oxide which enhances its positive effects on circulation [49].

A randomized trial, examined the effect of low dose L Arginine on fetoplacental circulation and subsequently on fetal growth in preeclampsia patients vs control patients, shows improved fetal gain and placental circulation in terms of Doppler indices of the middle cerebral umbilical arteries [50]. Another systematic review of randomized trials assessing its effectiveness in both prevention of preeclampsia in hypertensive pregnant patients besides preventing complication of preeclampsia, this study shows that it may be effective for both purposes [51]. Another randomized trial showed the effectiveness of L Arginine in preventing preeclampsia in high risk patients as well as decreasing IUGR and Preterm labor ratios compared to placebo controlled group [52]. Exciting results were found when a study assessed its effectiveness in preventing preeclampsia and its complications in high risk teenage pregnancies, the results have showed its positive physiological effects on both maternal and fetal circulations [53]

\section{Folic acid}

Folic acid is recommended by ACOG and other professional societies during preconception care and prenatal care to prevent neonatal neural tube defects [54]. Recent studies showed a debate around the possible role of folic acid in the prevention of preeclampsia in high-risk patients [55-57]. A large study using the UK regional database showed that supplementation with folic acid significantly reduces the risk of Small for Gestational Age at birth but only when started before pregnancy, regardless of other risk factors [57]. Although further research is needed to define the dose and onset of starting therapy [57]. Another analysis of 215 pregnant women who were enrolled in a pilot study showed that the concentration of folic acid in maternal blood was significantly higher following folic acid supplementation (24.6ng/mL vs.11.8ng/ $\mathrm{mL}$ ). Besides, the rates of both preeclampsia (odds ratio $[\mathrm{OR}]=0.27$, 95\% confidence interval $[\mathrm{CI}]=0.09-0.76)$ and small for gestational age (SGA: $9.2 \%$ vs. $20.0 \%$; OR= $0.42,95 \% \mathrm{CI}=0.18-0.99$ ) were lower in the folic acid supplementation group than those in the control group [58]. Another longitudinal study of women who obtained routine oral folic acid supplementation (15mg) as a prophylactic therapy for persistent preeclampsia after the first trimester with those who did not obtain it [59]. Tests found that women with previous preeclampsia who obtained routine supplementation of folic acid have a substantially decreased occurrence of overall preeclampsia, severe preeclampsia and early preeclampsia [59]. Another broad cohort study that involved a total of 10,041 pregnant women without recurrent hypertension or gestational hypertension was performed in 2010-2012 at the Gansu Provincial Maternity \& Child Care Hospital in Lanzhou, China. Researchers observed that consumers with folic acid supplementation have lower preeclampsia incidence relative to non-user groups $(\mathrm{OR}=$ $0.61,95 \% \mathrm{CI}=0.43-0.87)$. The reduced risk associated with Folic acid supplement was similar for mild or severe preeclampsia and early or late preeclampsia, although statistically significant associations were only observed for mild preeclampsia ( $\mathrm{OR}=0.50$, $95 \% \mathrm{CI}=0.30-0.81)$ and late onset $(\mathrm{OR}=0.60,95 \% \mathrm{CI}=0.42-0.86)$. The decreased risk correlated with dietary consumption of folate during pregnancy was shown only with severe preeclampsia (OR $=0.52,95 \% \mathrm{CI}=0.31-0.87$ ) [60]. Most studies agreed that further studies are needed to confirm this relationship and explain possible pathogenesis and mechanism of this preventive effect [59-62].

\section{Statins}

As preeclampsia bears pathogenic similarities with adult cardiovascular disorders such as endothelial dysfunction and inflammation that are central to both atherosclerosis and preeclampsia initiation and progression [63]. Moreover, they share common basis of risk factors like hypertension, diabetes, and obesity [64]. In preclinical animal trials, evidence supports the potential involvement of Statins in reversing the endothelial and cardiovascular impairment triggered by preeclampsia and reverses the angiogenesis imbalance induced by preeclampsia, contributing to a decrease in the risk of pregnancy loss and fetal growth restriction $[65,66]$. During the first phase of the clinical trial, Statins did not show any notable side effects in patients at high risk of developing preeclampsia with potential beneficial effects in terms of preeclampsia incidence reduction, premature labor, admission to neonatal care and improvement of the angiogenesis profile [63].

A review of 4 preterm preeclampsia patients who presented at $<30$ weeks of gestation and obtained Pravastatin every day. Pravastatin tended to regulate blood pressure, proteinuria and amounts of uric acid in the serum. In comparison, amounts of serum soluble forms such as tyrosine kinase -1 (sFlt-1) drop. They also collected the placenta at delivery and found that sFlt-1 secretion decreased by Pravastatin. These results show that Pravastatin reduced sFlt-1 and the production of soluble endoglin and decreased endothelial dysfunction in primary human tissues(67). Another study examined 21 pregnant women with an Antiphospholipid syndrome who experienced preeclampsia and/or restriction of intrauterine growth (IUGR) during low-dose aspirin (LDA) and low molecular weight heparin care. Low dose Aspirin and LMWH were given to the control group of 10 patients. 11 patients received Pravastatin $(20 \mathrm{mg} / \mathrm{d})$, in addition to LDA+LMWH. Hemodynamic of uteroplacental plasma, development of preeclampsia (hypertension 
and proteinuria), and fetal / neonatal outcomes is evaluated. Patients receiving Pravastatin and LDA+LMWH showed increased placental blood flow and improved preeclampsia features [68].

\section{Other lines of prevention}

Interventions in diet and lifestyles have the potential to reduce the risk of preeclampsia. While, the impact of additional therapeutic treatments on preeclampsia is uncertain in women with gestational diabetes mellitus [69]. Additionally, there was a decreased chance of preeclampsia with higher rates of physical activity before and during pregnancy [70]. Recombinant endothelial growth factor injection was found to be beneficial in preventing restriction of fetal development in animal models [71]. Metformin reduced sFlt-1 and sENG secretion from primary human tissues by potentially inhibiting the chain of transportation of mitochondrial electrons. In preterm preeclampsia placenta, the activity of the mitochondrial electron transport chain increased. Metformin decreased endothelial damage, increased omental vasodilatation and angiogenesis [72]. Methylprednisolone was not effective in maintaining platelet counts above 100,000 in preeclampsia patients with platelet count around 150,000 [73].

\section{Summary}

Hypertensive disorders of pregnancy are among the most common medical problems during pregnancy and they are associated with significant mortality and morbidity rate. Low dose Aspirin is already approved by many societies like ACOG and WHO to be used as prophylaxis for preeclampsia in high-risk patients. Recent studies showed a possible reduction in the incidence of preeclampsia and intrauterine growth restriction for high-risk mothers who taking LMWH during pregnancy. Although, the published evidence supporting LMWH is characterized by profound heterogeneity and inconsistency in terms of selection criteria and treatment regimens. Antepartum treatment with a combination of LMWH with low-dose ASA is endorsed by the American College of Chest Physicians and The American College of Obstetricians and Gynecologists for treatment of Antiphospholipid syndrome during pregnancy. WHO recommends Calcium as the first nutritional supplementation to prevent preeclampsia among population with low calcium in the diet? Folic acid, statins, and Arginine showed a possible reduction in incidence of preeclampsia in high-risk patients but there is a need for further studies to confirm that. Dietary and lifestyle interventions have the potential to reduce the risk of preeclampsia. Both Metformin and vascular endothelial growth factors has promising preventive role that has been found through recent studies.

\section{Acknowledgement}

None.

\section{Conflict of Interest}

Authors declare no conflict of interest.

\section{References}

1. Leeman Lawrence FP (2008) Hypertensive disorders of pregnancy. Am Acad Fam Physicians 35(2): 47-50.

2. Yu HF, Rao N, Dhillon IS (2015) Screening for Preeclampsia US Preventive Services Task Force Recommendation Statement 317(16): 1661-1667.

3. Amelia LM, Sutton MD, Lorie M. Harper MD, Alan TN, et al. (2018) Hypertensive Disorders in Pregnancy. Obstet Gynecol Clin North Am 45(2): 333-347.

4. Ganzevoort W, Rep A, de Vries JIP, Bonsel GJ, Wolf H (2006) Prediction of maternal complications and adverse infant outcome at admission for temporizing management of early-onset severe hypertensive disorders of pregnancy. Am J Obstet Gynecol 195(2): 495-503.

5. Lo JO, Mission JF, Caughey AB (2013) Hypertensive disease of pregnancy and maternal mortality. Curr Opin Obstet Gynecol 25(2): 124-132.

6. Cruz Barbosa IR, Merencio Silva WB, Gutierrez Cerqueira GS, Novo NF, Almeida FA, et al. (2015) Maternal and fetal outcome in women with hypertensive disorders of pregnancy: The impact of prenatal care. Ther Adv Cardiovasc Dis 9(4): 140-146.

7. Dadelszen P Von, Sawchuck D, Mcmaster R (2010) The Active Implementation of Pregnancy hypertension guidelines in British Columbia. Obstet Gynecol 116(3): 659-666

8. Menzies J, Magee LA, Li J, MacNab YC, Yin R, et al. (2007) Instituting surveillance guidelines and adverse outcomes in preeclampsia. Obstet Gynecol 110(1): 121-127.

9. Ankumah NE, Sibai BM (2017) Chronic Hypertension in pregnancy: diagnosis and management. Clin Obstet Gynecol 60: 206-214.

10. ACOG. (2002) ACOG practice bulletin 2002 Diagnosis and Management of Preeclampsia and Eclampsia. IJOG: (4).

11.Zuleta-Tobón JJ, Pandales-Pérez H, Sánchez S, Vélez-Álvarez GA, Velásquez-Penagos JA, et al. (2012) Hypertensive disorders of pregnancy. Am J Obstet Gynecol 7(2): 1581-1594.

12. Sibai BM (1996) Diagnosis and Management of Gestational Hypertension and Preeclampsia. ACOG, Elsevier 41(8): 67-78.

13. Lowe SA, Bowyer L, Lust K, McMahon LP, Morton M, et al. (2015) SOMANZ Guidelines for the Management of Hypertensive Disorders of Pregnancy 2014. Aust N Z J Obstet Gynaecol 55(5): e1-29.

14. James RM, Maurice D, Phyllis AA, Robert GR, George B, et al. (2013) Report of the American College of Obstetricians and Gynecologists. Task Force on Hypertension in Pregnancy 122(5): 1-100.

15. (2018) ACOG Committee Opinion Low-Dose Aspirin Use during Pregnancy. Obstet Gynecol 132(1): 44-52.

16. Correa PJ, Palmeiro Y, Soto MJ, Ugarte C, Illanes SE (2016) Etiopathogenesis, prediction, and prevention of preeclampsia. Hypertens Pregnancy 35(3): 280-294.

17. Phipps E, Prasanna D, Brima W, Jim B (2016) Preeclampsia: Updates in pathogenesis, definitions, and guidelines. Clin J Am Soc Nephrol 11(6): 1102-1113.

18. Jim B, Sharma S, Kebede T, Acharya A (2010) Hypertension in pregnancy: A comprehensive update. Cardiol Rev 18(4): 178-189.

19. WHO (2011) WHO recommendations for Prevention and treatment of pre-eclampsia and eclampsia. World Health Organization.

20. Redman CWG (2011) Hypertension in pregnancy: the NICE guidelines. Heart 97(23): 1967-1969.

21. Vest AR (2012) Hypertension in Pregnancy. Cardiology Clinics 30(3): 407-423.

22. Jim B, Karumanchi SA (2017) Preeclampsia: Pathogenesis, Prevention, and Long-Term Complications. Semin Nephrol 37(4): 386-397.

23. Dhariwal NK, Lynde GC (2017) Update in the Management of Patients with Preeclampsia. Anesthesiol Clin 35(1): 95-106. 
24. El-Sayed AAF (2017) Preeclampsia: A review of the pathogenesis and possible management strategies based on its pathophysiological derangements. Taiwan J Obstet Gynecol 56(5): 593-598.

25. Rosser ML, Katz NT (2013) Preeclampsia: An Obstetrician's Perspective. Adv Chronic Kidney Dis 20(3): 287-296.

26. Sonkusare S (2008) Hyperemesis gravidarum: A review. Med J Malaysia 63(3): 272-276.

27. Lee NM, Saha S (2011) Nausea and Vomiting of Pregnancy. Gastroenterol Clin north Am 40(2): 1-27.

28. Haram K, Mortensen JH, Nagy B (2014) Genetic aspects of preeclampsia and the HELLP syndrome. J Pregnancy 2014: 910751.

29. Chen CP (2009) Placental abnormalities and preeclampsla in trisomy 13 pregnancies. Taiwan J Obstet Gynecol 48(1): 3-8.

30. Heydanus R, Defoort P, Dhont M (1995) Pre-eclampsia and trisomy 13. Eur J Obstet Gynecol Reprod Biol 60(2): 201-202.

31. Bdolah Y, Palomaki GE, Yaron Y, Bdolah-Abram T, Goldman M, et al (2006) Circulating angiogenic proteins in trisomy 13. Am J Obstet Gynecol 194(1): 239-245.

32. Groom KM, McCowan LM, Mackay LK, Lee AC, Said JM, et al. (2017) Enoxaparin for the prevention of preeclampsia and intrauterine growth restriction in women with a history: a randomized trial. Am J Obstet Gynecol 216(3): 296.e1-296.e14.

33. Bergqvist D (2004) Low molecular weight heparin for the prevention of venous thromboembolism after abdominal surgery. Br J Surg 91(8): 965-974.

34. McLaughlin K, Baczyk D, Potts A, Hladunewich M, Parker JD, et al. (2017) Low Molecular Weight Heparin Improves Endothelial Function in Pregnant Women at High Risk of Preeclampsia. Hypertension 69(1): 180-188.

35. D’Ippolito S, Di Nicuolo F, Marana R, Castellani R, Stinson J, et al. (2012) Emerging nonanticoagulant role of low molecular weight heparins on extravillous trophoblast functions and on heparin binding-epiderma growth factor and cystein-rich angiogenic inducer 61 expression. Fertil Steril 98(4): 1028-1036.

36. Di Simone N, Di Nicuolo F, Sanguinetti M, Ferrazzani S, D’Alessio MC, et al. (2007) Low-molecular Weight Heparin Induces In Vitro Trophoblast Invasiveness: Role of Matrix Metalloproteinases and Tissue Inhibitors. Placenta 28(4): 298-304

37. Hills FA, Abrahams VM, González-Timón B, Francis J, Cloke B, et al (2006) Heparin prevents programmed cell death in human trophoblast. Mol Hum Reprod 12(4): 237-243.

38. Sotiriadis A (2016) Low-dose aspirin plus low-molecular-weight heparin for the prevention of pre-eclampsia: Yes, no or maybe. Ultrasound Obstet Gynecol 47(5): 539-541.

39. Roberge S, Demers S, Nicolaides KH, Bureau M, Côté S, Bujold E (2016) Prevention of pre-eclampsia by low-molecular-weight heparin in addition to aspirin: A meta-analysis. Ultrasound Obstet Gynecol 47(5): 548-553.

40. Rodger MA, Hague WM, Kingdom J, Kahn SR, Karovitch A, et al. (2014) Antepartum dalteparin versus no antepartum dalteparin for the prevention of pregnancy complications in pregnant women with thrombophilia (TIPPS): A multinational open-label randomised trial. Lancet 384(9955): 1673-1683.

41. Katsi V, Kanellopoulou T, Makris T, Nihoyannopoulos P, Nomikou E, et al. (2016) Aspirin vs. Heparin for the Prevention of Preeclampsia. Curr Hypertens Rep 18(7): 57

42. Countries L, Stoltzfus RJ (2016) Calcium Supplementation to Prevent Preeclampsia: Translating Guidelines into Practice. Am Soc Nutr 7(1): 275-278.

43. The Seventh Report of the Joint National Committee on Prevention, Detection, Evaluation, and Treatment of High Blood Pressure,Complete
Report The Seventh Report of the Joint National Committee on Prevention, Detection, Evaluation, and Treatment of High $\mathrm{Bl}$

44. Brown MA, Magee LA, Kenny LC, Karumanchi SA, McCarthy FP, et al. (2018) Hypertensive disorders of pregnancy: ISSHP classification, diagnosis, and management recommendations for international practice. Hypertension 72(1): 24-43.

45. (2013) World Health Organization. Guideline : Calcium supplementation in pregnant women. World Heal Organ: 24.

46. IOM (2010) Dietary Reference Intakes for Calcium and Vitamin D Health Effects of Vitamin D and Calcium Intake. Inst Med das Acad Nac: 1-4.

47. Hanzlik RP, Fowler SC, Fisher DH (2005) Relative Bioavailability of Calcium from Calcium Formate, Calcium Citrate, and Calcium Carbonate. J Pharmacol Exp Ther 313(3): 1217-1222.

48. Savvidou MD, Hingorani AD, Tsikas D, Frölich JC, Vallance P, et al. (2003) Endothelial dysfunction and raised plasma concentrations of asymmetric dimethylarginine in pregnant women who subsequently develop pre-eclampsia. Lancet 361(9368): 1511-1517.

49. Böger RH (2018) Asymmetric Dimethylarginine, an Endogenous Inhibitor of Nitric Oxide Synthase, Explains the "L-Arginine Paradox" and Acts as a Novel Cardiovascular Risk Factor. J Nutr 134(10 Suppl): 2842S-2847S

50. Rytlewski K, Olszanecki R, Lauterbach R, Grzyb A, Basta A (2006) Effects of oral L-arginine on the foetal condition and neonatal outcome in preeclampsia: A preliminary report. Basic Clin Pharmacol Toxicol 99(2): 146-152.

51. Dorniak-Wall T, Grivell RM, Dekker GA, Hague W, Dodd JM (2014) The role of L-arginine in the prevention and treatment of pre-eclampsia: A systematic review of randomised trials. J Hum Hypertens 28(4): 230235.

52. Camarena Pulido EE, García Benavides L, Panduro Barón JG, Pascoe Gonzalez S, Madrigal Saray AJ, et al. (2016) Efficacy of L-arginine for preventing preeclampsia in high-risk pregnancies: A double-blind, randomized, clinical trial. Hypertens Pregnancy 35(2): 217-225.

53. Razik MA, El-Berry S, Abosereah M, Edris Y, Sharafeldeen A (2016) Prophylactic treatment for preeclampsia in high-risk teenage primigravidae with nitric oxide donors: A pilot study. J Matern Neonatal Med 29(16): 2617-2620.

54. Cheschier N (2003) ACOG Practice Bulletin. Neural Tube Defects. Number 44, July 2003. (Replaces Committee Opinion Number 252, March 2001). Int J Gynaecol Obstet 83(1): 123-123.

55. Thériault S, Giguère Y, Massé J, Lavoie SB, Girouard J, et al. (2013) Absence of Association Between Serum Folate and Preeclampsia in Women Exposed to Food Fortification. Obstet Gynecol 122(2 Pt 1):345351

56. Li Z, Ye R, Zhang L, Li H, Liu J, et al. (2013) Folic acid supplementation during early pregnancy and the risk of gestational hypertension and preeclampsia. Hypertension 61(4): 873-879.

57. Hodgetts VA, Morris RK, Francis A, Gardosi J, Ismail KM (2015) Effectiveness of folic acid supplementation in pregnancy on reducing the risk of small-for-gestational age neonates: A population study, systematic review and meta-analysis. BJOG An Int J Obstet Gynaecol 122(4): 478-490.

58. Kim MW, Ahn KH, Ryu KJ, Hong SC, Lee JS, et al. (2014) Preventive effects of folic acid supplementation on adverse maternal and fetal outcomes. PLoS One 9(5): 1-5.

59. Saccone G, Sarno L, Roman A, Donadono V, Maruotti GM, et al. (2016) 5 -Methyl-tetrahydrofolate in prevention of recurrent preeclampsia. J Matern Neonatal Med 29(6): 916-920.

60. Wang Y, Zhao N, Qiu J, He X, Zhou M, et al. (2015) Folic acid supplementation and dietary folate intake, and risk of preeclampsia. Eur J Clin Nutr 69(10): 1145-1150. 
61. Mantovani E, Filippini F, Bortolus R, Franchi M (2014) Folic acid supplementation and preterm birth: Results from observational studies. Biomed Res Int 2014: 481914

62. Wen SW, Walker M (2010) Effect of folic acid supplementation in pregnancy on preeclampsia. Pregnancy Hypertens An Int J Women's Cardiovasc Heal 1: S28.

63. Costantine MM, Cleary K, Hebert MF, Ahmed MS, Brown LM, et al. (2017) Safety and Pharmacokinetics of Pravastatin Used for the Prevention of Preeclampsia in High-Risk Pregnant Women: A Pilot Randomized Controlled Trial.

64. Barton JR, Sibai BM (2008) Clinical Expert Series Prediction and Prevention of Recurrent Preeclampsia. Obstet Gynecol 112(2): 359-372.

65. Cudmore M, Ahmad S, Al-Ani B, Fujisawa T, Coxall H, et al. (2007) Negative regulation of soluble Flt-1 and soluble endoglin release by heme oxygenase-1. Circulation 115(13): 1789-1797.

66. Costantine MM, Tamayo E, Lu F, Bytautiene E, Longo M, et al. (2010) Using pravastatin to improve the vascular reactivity in a mouse model of soluble Fms-like tyrosine kinase-1-induced preeclampsia. Obstet Gynecol 116(1): 114-120.

67. Brownfoot FC, Tong S, Hannan NJ, Binder NK, Walker SP, et al. (2015) Effects of Pravastatin on Human Placenta, Endothelium, and Women with Severe Preeclampsia. Hypertension 66(3): 687-697.
68. Lefkou E, Mamopoulos A, Dagklis T, Vosnakis C, Rousso D, et al. (2016) Pravastatin improves pregnancy outcomes in obstetric antiphospholipid syndrome refractory to antithrombotic therapy. J Clin Invest 126(8): 2933-2940.

69. Allen R, Rogozinska E, Sivarajasingam P, Khan KS, Thangaratinam S (2014) Effect of diet- And lifestyle-based metabolic risk-modifying interventions on preeclampsia: A meta-analysis. Acta Obstet Gynecol Scand 93(10): 973-985.

70. Aune D, Saugstad OD, Henriksen T, Tonstad S (2014) Physical Activity and the Risk of Preeclampsia. Epidemiology 25(3): 331-343.

71. Sulistyowati S, Bachnas MA, Anggraini ND, Yuliantara EE, Prabowo W, et al. (2017) Recombinant vascular endothelial growth factor 121 injection for the prevention of fetal growth restriction in a preeclampsia mouse model. J Perinat Med 45(2): 245-251.

72. Brownfoot FC, Hastie R, Hannan NJ, Cannon P, Tuohey L, et al. (2016) Metformin as a prevention and treatment for preeclampsia: Effects on soluble fms-like tyrosine kinase 1 and soluble endoglin secretion and endothelial dysfunction. Am J Obstet Gynecol 214(3): 356.

73. Pourrat O, Dorey M, Ragot S, De Hauteclocque A, Deruelle, et al. (2016) High-Dose Methylprednisolone to Prevent Platelet Decline in Preeclampsia: A Randomized Controlled Trial. Obstet Gynecol 128(1): 153-158. 